\title{
Actividades de conservación y de educación ambiental en torno a una especie emblemática, el tapir de montaña (Tapirus pinchaque), en sectores andinos del sur de Ecuador
}

\section{Environmental conservation and education activities around an emblematic species, the mountain tapir (Tapirus pinchaque), in the Andean sectors of southern Ecuador}

\author{
Pedro Álvarez \\ Universidad Internacional del Ecuador, Ecuador \\ Patricio Yánez M. \\ Universidad Internacional del Ecuador, Ecuador \\ Universidad Iberoamericana del Ecuador-UNIB.E, Ecuador \\ Autor para correspondencia: pedrojalvarez@yahoo.com \\ Fecha de recepción: 01 de Febrero de 2017 - Fecha de aceptación: 10 de Agosto de 2017
}

Resumen: la Universidad Internacional del Ecuador (Sede Loja) en conjunto con la Fundación de Conservación Jocotoco, desde agosto de 2015, vienen desarrollando actividades de cuidado del tapir de montaña (Tapirus pinchaque) y los hábitats en los que se desarrolla en Los Andes del sur de Ecuador, en ambientes de bosque nublado y páramos de la Reserva Biológica Tapichalaca y zonas colindantes. Para ello se propuso la realización del presente proyecto que busca establecer un sistema de monitoreo e investigación de esta especie bandera con fines ecoturísticos, así como también para apoyar la capacitación en educación ambiental; mediante un diagnóstico preliminar y la implementación de un sistema de investigación y monitoreo de los especímenes mediante cámaras trampa y observación directa, con el fin de generar datos poblacionales e imágenes de esta especie en su hábitat natural e identificar los sitios más idóneos para observar estos animales en actividades de ecoturismo; se busca además crear cartillas y cuentos didácticos que describan los principales aspectos ecológicos del tapir de montaña, sin descuidar el desarrollo de propuestas de conservación, acordes con los objetivos del milenio, las metas estratégicas de los gobiernos autónomos descentralizados parroquiales y provinciales locales y los Planes de Manejo de los Parques Nacionales Podocarpus y Yacuri.

Palabras clave: conservación biológica; educación ambiental; especie bandera; tapir de montaña; tapirus pinchaque

Abstract: The Universidad Internacional del Ecuador (Loja), together with the Jocotoco Conservation Foundation, since August 2015, have been carrying out activities to take care of the mountain tapir (Tapirus pinchaque) and the habitats where this species lives in the southern Andes of Ecuador, in environments of cloud forests and páramos of the Biological Reserve Tapichalaca and adjoining areas. For this, we proposed to carry out the present project that seeks to establish a system for monitoring and research this emblematic species for ecotourism purposes, as well as to support the training in environmental education. A preliminary diagnosis and the implementation 
of a system of investigation and monitoring using trap cameras and direct observation is carrying out, in order to generate population data and images of this species in its natural habitat and identify the most suitable sites to observe it in Ecotourism activities. It is also sought to create primers and didactic stories that describe the main ecological aspects of the mountain tapir. Finally, conservation proposals are drawn up to achieve a sustainable development of the activity, in accordance with the millennium goals, and the strategic goals of the autonomous decentralized parish and provincial governments as well as the Management Plans of the Podocarpus and Yacuri National Parks.

Key words: biological conservation; environmental education; flag species; mountain tapir; tapirus pinchaque

\section{Introducción}

La Universidad Internacional del Ecuador (Extensión Loja) a través de la Escuela de Gestión Turística y Medio Ambiente, consciente de las oportunidades que representan los recursos turísticos naturales y el respeto a la biodiversidad ha emprendido una serie de acciones para precautelar especies emblemáticas y los ambientes en los que se desarrollan.

Entre ellas, se propuso la realización del presente proyecto con la finalidad de establecer un sistema de monitoreo e investigación del tapir de montaña (Tapirus pinchaque; Figura 1). La observación de esta especie en su hábitat natural está empezando a ser realizada por empresas de orientación ecoturística.

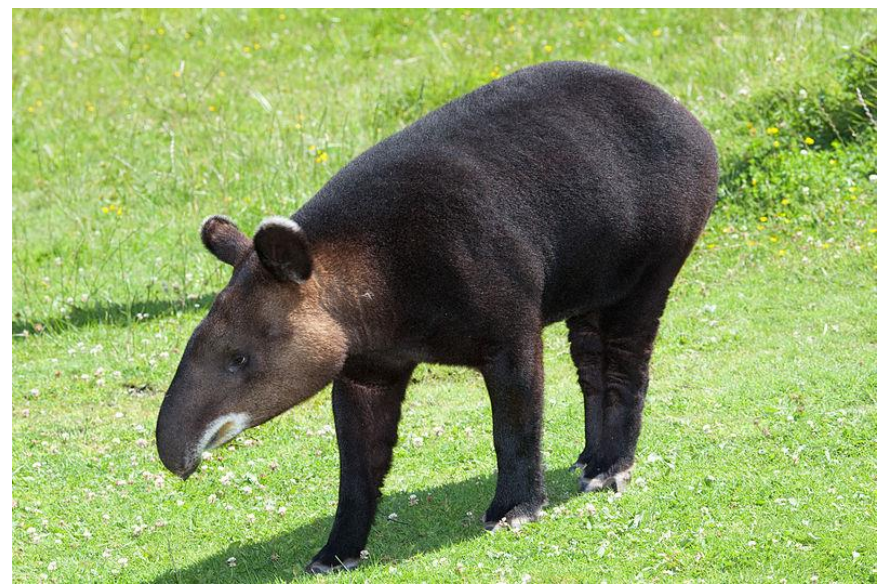

Figura 1. Individuo adulto del tapir de montaña (Tapirus pinchaque Roulin)

Fuente: https://upload.wikimedia.org/

Igualmente, al desarrollar el presente proyecto se busca fortalecer actividades de capacitación y educación ambiental dirigidas a habitantes locales, partiendo de un diagnóstico preliminar de la situación poblacional de esta especie y la implementación de un sistema de investigación y monitoreo de sus especímenes en sus ambientes naturales de vida, tal como lo recomiendan Castellanos (1994), Brooks \& Eisenberg (1999), Bermudez (2010), Correa \& Torres (2005).

A la vez, se buscan identificar los sitios más idóneos para efectuar ecoturismo y poder observar ejemplares de esta especie, así como la elaboración de una cartilla o cuento didáctico 
que describa en términos sencillos los aspectos ecológicos más relevantes de la vida del tapir de montaña.

Las actividades anteriores permitirían delinear propuestas más amplias de conservación de los bosques nublados y páramos del sur del Ecuador, en dónde se tengan reportes de avistamientos del tapir de montaña, buscando a la vez un desarrollo sustentable de la actividad ecoturística local, acorde con los objetivos del Plan Nacional del Buen Vivir 2012-2017 (Senplades, 2012), los PDOT parroquiales y provinciales; y los Planes de Manejo del Parque Nacional Podocarpus y del Parque Nacional Yacuri (http://areasprotegidas.ambiente.gob.ec/es/documentos).

\section{Materiales y métodos}

\section{Área de estudio}

La presente investigación fue desarrollada en los Andes del sur del Ecuador en ambientes de bosque nublado y páramos de la Reserva Biológica Tapichalaca (Figura 2) ubicados en la Parroquia Valladolid, Cantón Palanda, Provincia Zamora Chinchipe.

El territorio parroquial de Valladolid limita al norte con la parroquia Yangana de la provincia de Loja, en la cordillera de Sabanillas; al sur con la parroquia Palanda hasta la quebrada de Pueblo Viejo, al este con la parroquia El Porvenir del Carmen en el Río Numbala y al oeste con el cantón Espíndola de la provincia de Loja.

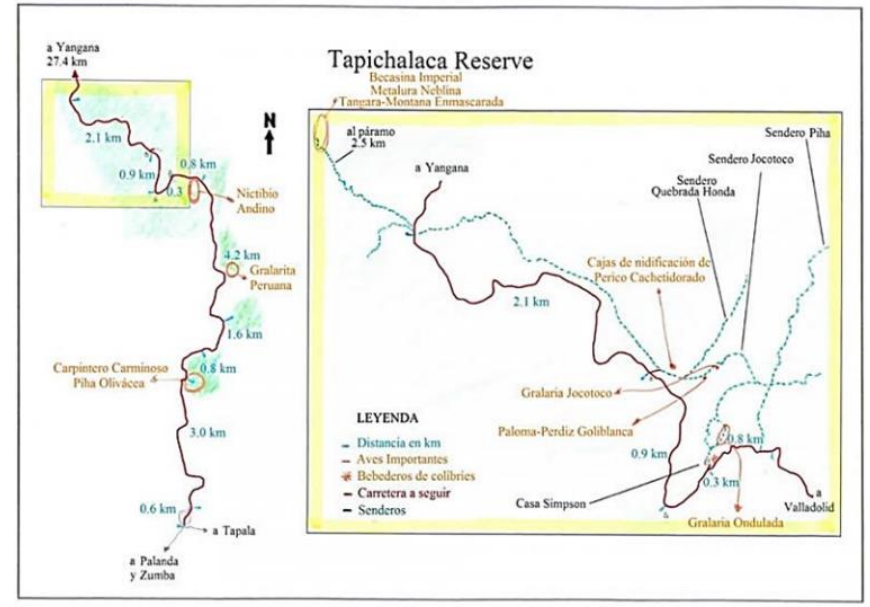

Figura 2. Ubicación del área de estudio (ambientes entre Valladolid y Yangana).

Fuente: cortesía de la Fundación de Conservación Jocotoco

El área de estudio fue elegida ya que cuenta con 4 sitios idóneos para desarrollar ecoturismo, centrado en la observación de mamíferos grandes (oso de anteojos, Tremarctos ornatus; tapir de montaña, Tapirus pinchaque; lobo o zorro de páramo, Lycalopex culpaeus) y también de aves de páramo (gallinago imperial, Gallinago imperialis; colibrí gigante, Patagona gigas; y otras (Anexo 1)). 
Igualmente, puede constituirse en una zona de observación del cambio de formaciones vegetales desde ambientes de bosque nublado hasta los de páramo (sensu Yánez, 1997a; Yánez, 1997b) e incluso en una zona de monitoreo de la respuesta de las especies vegetales a eventos de cambio climático (sensu Yánez \& Fariñas, 2000; Yánez \& Picoíta, 2012).

\section{Caracterización del recurso turístico}

Se ha iniciado el levantamiento de información de inventario de atractivos turísticos de acuerdo a lo que recomiendan autores como Nasimba y Cejas (2015), tomando en cuenta las especificaciones técnicas descritas para el registro fotográfico, tarea a cargo de algunos docentes y estudiantes de la Escuela de Turismo de la UIDE. Tal información está siendo procesada a la presente fecha.

\section{Implementación de las cámaras trampa}

Las cámaras trampa funcionales fueron ubicadas en cuatro zonas diferentes de monitoreo, correspondientes a bosque nublado (2), páramo (1) y riveras de un sistema lacustre paramero (1). Estos dispositivos nos permitieron registrar información sobre la ecología y abundancia de los tapires, e indirectamente información sobre sitios claves para el desarrollo del ecoturismo para observación de esta especie bandera y de otras típicas de estas zonas andinas.

\section{Resultados y discusión}

Se determinaron, a través del uso de 37 fichas semanales de monitoreo del tapir andino (Tapirus pinchaque) cuatro sitios idóneos para desarrollar ecoturismo de observación de fauna y flora, centrado en la observación de mamíferos grandes.

Se obtuvo un banco de fotografías de doce meses de investigación y un banco de videos de ocho meses, mismos que a la presente fecha se están procesando.

\section{Registros de abundancia del tapir de montaña}

En la Tabla 1 se puede observar el número de registros de presencia de tapir de montaña en los 4 sitios de monitoreo, a lo largo de los doce meses de estudio.

Tabla No. 1. Registros de presencia de tapir de montaña (por observación directa de ejemplares y observación indirecta: huellas, rastros) en cuatro sitios de observación en el presente estudio

Código del sitio

\begin{tabular}{lcccc} 
Mes de registro & $\begin{array}{c}\text { Bosque } \\
\text { nublado } \\
\text { BN1 }\end{array}$ & $\begin{array}{c}\text { Bosque } \\
\text { nublado } \\
\text { BN2 }\end{array}$ & $\begin{array}{c}\text { Páramo } \\
\text { P1 }\end{array}$ & $\begin{array}{c}\text { Laguna de } \\
\text { páramo } \\
\text { LP1 }\end{array}$ \\
\hline sep 2015 & & & & \\
oct 2015 & 0 & 0 & 2 & 5 \\
\hline
\end{tabular}




\begin{tabular}{lcccc}
\hline nov 2015 & 0 & 0 & 0 & 4 \\
dic 2015 & 2 & 4 & 0 & 6 \\
ene 2016 & 8 & 10 & 2 & 4 \\
feb 2016 & 10 & 4 & 1 & 0 \\
mar 2016 & 12 & 8 & 0 & 2 \\
abr 2016 & 8 & 12 & 1 & 0 \\
may 2016 & 4 & 6 & 0 & 1 \\
jun 2016 & 2 & 2 & 0 & 2 \\
jul 2016 & 2 & 4 & 2 & 4 \\
ago 2016 & 2 & 2 & 2 & 2 \\
Total de registros de presencia del tapir de montaña & 50 & 52 & 10 & 36 \\
Promedio de registros por mes & 4,17 & 4,33 & 0,83 & 3,00 \\
Mediana de registros por mes & 2,00 & 4,00 & 0,50 & 3,00 \\
\hline
\end{tabular}

Con los registros de observación de los cuatro sitios se corrió un Análisis de Varianza no Paramétrico (Kruskal-Wallis), de acuerdo a lo recomendado por Yánez (2005) cuando no se detecta distribución normal en los juegos de datos a ser comparados. El estadístico H fue de 8,87; $\mathrm{p}=0,03$. Esto permitió distinguir que los sitios BN1, BN2 y LP1 son significativamente diferentes a P1.

BN1, BN2 y LP1 son sitios en los que la probabilidad de observar tapires de montaña es mayor a lo largo del año, y constituirían, al menos de manera preliminar, sitios en los cuales se podría realizar la observación de este tipo de fauna en la zona de estudio a través de actividades de ecoturismo planificadas (sensu Conservation Tourism, 2010), considerando el avistamiento a distancias razonables y con el uso de largavistas para no causar ningún tipo de stress en estos animales, tal como lo recomiendan algunos expertos en la conservación del tapir de montaña (Downer, 1996; Downer, 2001; Downer \& Castellanos, 2002).

\section{Recomendaciones}

Se recomienda que se continúe recabando información de aspectos ecológicos poblacionales y ecosistémicos de Tapirus pinchaque en la zona de trabajo y áreas contiguas, para avanzar con la iniciativa de desarrollar actividades de desarrollo ecoturístico en la zona.

Actividades de ecoturismo, turismo comunitario y de educación ambiental deben estar articuladas en un plan a mediano o largo plazo (Yánez, 2014), siempre aplicando el uso cuidadoso del recurso paisajístico andino local, y la consideración de la especie bandera Tapirus pinchaque, en torno a la cual giraría potencialmente la planificación del desarrollo de la actividad turística-educativa.

Asimismo, se pueden desarrollar en la zona actividades de capacitación para estudiantes universitarios en ciencias ambientales, mismas que girarían en torno a los beneficios de la conservación de ambientes naturales nativos y formas alternativas de uso de los recursos naturales con bajo impacto ambiental, tales como las de ecoturismo y agroturismo (DoumetChilán \& Yánez, 2014), turismo cultural (Nasimba \& Cejas, 2015), manejo de productos de especies nativas (Yánez, 2012b) entre otros. 


\section{Conclusiones}

En general, podemos mencionar que el tapir de montaña es una especie relativamente difícil de observar de manera directa, pero es un importante indicador de buena conservación de un hábitat andino, y a la vez puede atraer viajeros y turistas de naturaleza de diferentes partes del mundo.

En el presente estudio se han determinado cuatro zonas de avistamiento de tapires en la Reserva Tapichalaca, en tres de las cuales (dos de bosque nublado y una laguna paramera) se tiene una alta probabilidad de observación de estos animales a lo largo del año. El avistamiento de estos animales amerita cierta preparación física en los visitantes, y una capacidad media para efectuar trekking en ambientes montañosos. Por lo que se puede concluir que en los senderos locales se puede desarrollar ecoturismo y además un turismo alternativo o de aventura.

Se tienen bien registrados los datos de los sitios de avistamiento, esto permitirá sugerir al personal de la Reserva Tapichalaca que se los cuide y monitoree de manera planificada. La idea de desarrollar una cartilla y/o cuento didáctico para la zona, que gire en torno al tapir sigue en pie y se espera contar con un tesista universitario que desarrolle esta actividad en los meses próximos.

\section{Bibliografía}

\section{Fuentes impresas:}

Bermúdez, D. (2010). Dieta y hábitos alimenticios del Tapir Andino (T. pinchaque) entre localidades del Corredor Ecológico Llanganates - Sangay. Quito: Universidad Central del Ecuador, Tesis de Licenciatura.

Brooks, D., \& Eisenberg, J. (1999). Estado y biología de los tapires neotropicales: perspectiva general. Manejo y conservación de fauna silvestre en América Latina, 409-414.

Castellanos, A. (1994). El tapir andino (Tapirus pinchaque Roulin): crianza de un ejemplar en el Bosque Protector Pasochoa y notas ecológicas en el Parque Nacional Sangay, Ecuador. Quito: Universidad Central del Ecuador, Tesis de Licenciatura.

Correa, A., \& Torres, N. (2005). Estrategia nacional preliminar para la conservación de los tapires (género Tapirus) en el Ecuador. Loja: Universidad Técnica Particular de Loja, Tesis de Licenciatura.

Downer, C. (1996). The Mountain Tapir, endangered "flagship" species of the high Andes. Oryx, $30,45-58$.

Downer, C. (2001). Observations on the diet and habitat of the mountain tapir (Tapirus pinchaque). J. Zool. Lond., 254, 279-291. 
Downer, C., \& Castellanos, A. (2002). Tapirus pinchaque. En: IUCN Red List of Threatened Species.

Doumet-Chilán, Y., \& Yánez, P. (2014). Estrategias para desarrollar el agroturismo en la represa Sixto Durán Ballén, Manabí, Ecuador. ESPAMCIENCIA, 5(1), 7-15.

Nasimba, C., \& Cejas, M. (2015). Diseño de productos turísticos y sus facilidades. Qualitas, 10, 22-39.

Senplades. (2012). Plan Nacional del Buen Vivir. Quito.

Yánez, A. P. (1997a). Seminario Bibliográfico "Distribución de especies vegetales en ecosistemas naturales desde la óptica del Continuum: Evolución de la Idea y algunos Métodos para su Caracterización”. CIELAT, Universidad de Los Andes, Mérida, Venezuela, 44pp.

Yánez, A. P. (1997b). Análisis de la distribución de especies vegetales a lo largo de un gradiente altitudinal Páramo-Selva Nublada del Parque Nacional Sierra Nevada, Venezuela. Tesis de Maestría en Ecología Tropical. Universidad de Los Andes. Mérida, Venezuela.

Yánez, P., \& Fariñas, M. (2000). Estimación de óptimos de distribución de especies vegetales en un gradiente altitudinal andino y su relación con el monitoreo futuro de estos ambientes. Memorias del IV Congreso Latinoamericano de Ecología. Arequipa: UNSA.

Yánez, P. (2005). Biometría y Bioestadística fundamentales. Analizando la estructura numérica de la información en proyectos ecológicos. Quito.

Yánez, P., \& Picoíta, F. (2012). Posibles efectos del cambio climático global en ambientes andinos de bosque montano y páramo de la sierra centro de Ecuador. Quito: UNIBE.

Yánez, P. (2012b). Consideraciones para el diseño y aplicación de planes de manejo de especies vegetales silvestres no maderables de interés comercial. Qualitas, 4, 31-40.

Yánez, P. (2014). Un vistazo a la conservación Biológica del Ecuador. Qualitas, 7, 80-84.

\section{Fuentes virtuales:}

Conservation Tourism. (2010). Wallingford, GB: CAB International. Retrieved from http://www.ebrary.com

http://areasprotegidas.ambiente.gob.ec/es/documentos Fecha de consulta: 20 de diciembre 2016.

https://upload.wikimedia.org/wikipedia/commons/2/21/Tapirus_pinchaque_portrait.jpg Fecha de consulta: 15 de diciembre 2016.

IUCN. 2008. 2008 IUCN Red list of Theatered Species. www.Iucnredlist.org 
Anexo No. 1. Registros de aves efectuados en la zona de estudio en dos jornadas de observación en 2016.

\begin{tabular}{|c|c|c|c|c|}
\hline No. & $\begin{array}{l}\text { Nombre común } \\
\text { en español }\end{array}$ & $\begin{array}{c}\text { Nombre común en inglés y } \\
\text { nombre científico }\end{array}$ & $\begin{array}{r}\text { No. de registros } \\
\text { en bosque nublado }\end{array}$ & $\begin{array}{l}\text { No. de registros en } \\
\text { páramo arbustivo }\end{array}$ \\
\hline 1 & $\begin{array}{c}\text { Águila } \\
\text { pechinegra }\end{array}$ & $\begin{array}{l}\text { (Black-chested buzzard-eagle; } \\
\text { Geranoaetus melanoleucus) }\end{array}$ & 0 & 2 \\
\hline 2 & $\begin{array}{l}\text { Amazona } \\
\text { nuquiescamosa }\end{array}$ & $\begin{array}{l}\text { (Scaly-naped amazon; } \\
\text { Amazona mercenaria) }\end{array}$ & 6 & 4 \\
\hline 3 & $\begin{array}{l}\text { Barba blanca } \\
\text { rayada }\end{array}$ & $\begin{array}{l}\text { (Streaked tufttedchneek; } \\
\text { Pseudocolaptes boissonneautii) }\end{array}$ & 4 & 0 \\
\hline 4 & $\begin{array}{c}\text { Cacique } \\
\text { piquiamarillo }\end{array}$ & $\begin{array}{l}\text { (Yellow-billed cacique; } \\
\text { Amblycercus Holosericeus) }\end{array}$ & 3 & 0 \\
\hline 5 & $\begin{array}{r}\text { Canastero } \\
\text { multilistado }\end{array}$ & $\begin{array}{l}\text { (Many-striped canastero; } \\
\text { Asthenes flammulata) }\end{array}$ & 0 & 18 \\
\hline 6 & $\begin{array}{l}\text { Carpintero } \\
\text { poderoso }\end{array}$ & $\begin{array}{l}\text { (Powerful woodpecker; } \\
\text { Campeohilus pollens) }\end{array}$ & 2 & 0 \\
\hline 7 & Cerceta andina & $\begin{array}{l}\text { (Andean Teal; } \\
\text { Anas andium) }\end{array}$ & 0 & 8 \\
\hline 8 & $\begin{array}{l}\text { Cernícalo } \\
\text { americano }\end{array}$ & $\begin{array}{l}\text { (American kestrel; } \\
\text { Falco sparverius) }\end{array}$ & 2 & 0 \\
\hline 9 & $\begin{array}{l}\text { Chotacabras } \\
\text { alifajeado }\end{array}$ & $\begin{array}{l}\text { (Band-winged nighjar; } \\
\text { Caprimulgus longirostris) }\end{array}$ & 1 & 0 \\
\hline 10 & $\begin{array}{r}\text { Cinclodes } \\
\text { alifranjeado }\end{array}$ & $\begin{array}{l}\text { (Bar-winged cinclodes; } \\
\text { Cinclodes fuscus) }\end{array}$ & 0 & 8 \\
\hline 11 & $\begin{array}{l}\text { Colaespina de } \\
\text { azara }\end{array}$ & $\begin{array}{l}\text { (Azaras' spinetail; } \\
\text { Synallaxis azarae) }\end{array}$ & 16 & 4 \\
\hline 12 & Colibrí gigante & $\begin{array}{l}\text { (Giant hummingbird; } \\
\text { Patagona gigas) }\end{array}$ & 0 & 6 \\
\hline 13 & $\begin{array}{c}\text { Colibrí } \\
\text { terciopelo }\end{array}$ & $\begin{array}{l}\text { (Mountain velvet-breast; } \\
\text { Lafresnaya lafresnayi) }\end{array}$ & 6 & 0 \\
\hline 14 & $\begin{array}{l}\text { Colicardo } \\
\text { murino }\end{array}$ & $\begin{array}{l}\text { (Mouse-colored thistletail; } \\
\text { Schizoeaca griseomurina) }\end{array}$ & 4 & 22 \\
\hline 15 & Elanio tijereta & $\begin{array}{l}\text { (Swallow-tailed kite; } \\
\text { Elanoides forficatus) }\end{array}$ & 6 & 2 \\
\hline 16 & $\begin{array}{l}\text { Estrellita } \\
\text { ventriblanca }\end{array}$ & $\begin{array}{l}\text { (White-bellied woodstar; } \\
\text { Chaetocercus mulsant) }\end{array}$ & 5 & 0 \\
\hline 17 & $\begin{array}{l}\text { Gallinago } \\
\text { imperial }\end{array}$ & $\begin{array}{l}\text { (Imperial Snipe; } \\
\text { Gallinago imperialis) }\end{array}$ & 0 & 12 \\
\hline 18 & Gorridiadema & $\begin{array}{l}\text { (Plushcap; } \\
\text { Catamblyrhychus diadema) }\end{array}$ & 4 & 0 \\
\hline 19 & $\begin{array}{l}\text { Grallaria } \\
\text { leonada }\end{array}$ & $\begin{array}{l}\text { (Tawny antpitta; } \\
\text { Grallaria quitensis) }\end{array}$ & 0 & 8 \\
\hline 20 & Grallaria rufula & $\begin{array}{l}\text { (Rufous antpittta; } \\
\text { Grallaria rufula) }\end{array}$ & 6 & 0 \\
\hline 21 & Inca collarejo & $\begin{array}{l}\text { (Collared inca; } \\
\text { Coeligena torquata) }\end{array}$ & 12 & 0 \\
\hline 22 & $\begin{array}{l}\text { Jilguero } \\
\text { encapuchado }\end{array}$ & $\begin{array}{l}\text { (Hooded siskin; } \\
\text { Carduelis magellanica) }\end{array}$ & 4 & 0 \\
\hline 23 & Metalura tiria & $\begin{array}{l}\text { (Tyrian metaltail; } \\
\text { Metallura tyrianthina) }\end{array}$ & 6 & 0 \\
\hline 24 & Mirlo grande & $\begin{array}{l}\text { (Great thrush; } \\
\text { Turdus fuscater) }\end{array}$ & 20 & 0 \\
\hline 25 & Paloma callareja & $\begin{array}{l}\text { (Band tailed pijeon; } \\
\text { Columba fasciata) }\end{array}$ & 20 & 0 \\
\hline
\end{tabular}




\begin{tabular}{|c|c|c|c|c|}
\hline 26 & Pava barbada & $\begin{array}{l}\text { (Bearded guan; } \\
\text { Penelope barbata) }\end{array}$ & 12 & 0 \\
\hline 27 & $\begin{array}{l}\text { Perico } \\
\text { cacheidorado }\end{array}$ & $\begin{array}{l}\text { (Golden-plumed parakeet; } \\
\text { Leptosittaca branickii) }\end{array}$ & 8 & 0 \\
\hline 28 & $\begin{array}{l}\text { Pinchaflor } \\
\text { enmascarado }\end{array}$ & $\begin{array}{l}\text { (Masked flowerpiecer; } \\
\text { Diglossopis cyanea) }\end{array}$ & 12 & 0 \\
\hline 29 & $\begin{array}{l}\text { Pinchaflor } \\
\text { flanquiblanco }\end{array}$ & $\begin{array}{l}\text { (White-sided flower-piercer; } \\
\text { Diglossa albilatera) }\end{array}$ & 10 & 0 \\
\hline 30 & Pinchaflor negro & $\begin{array}{l}\text { (Black flowerpiecer; } \\
\text { Diglossa humeralis) }\end{array}$ & 12 & 0 \\
\hline 31 & $\begin{array}{l}\text { Semillero } \\
\text { paramelo }\end{array}$ & $\begin{array}{l}\text { (Paramo seedeater; } \\
\text { Catamenia homochroa) }\end{array}$ & 0 & 4 \\
\hline 32 & $\begin{array}{l}\text { Tangara } \\
\text { montana } \\
\text { lagrimosa }\end{array}$ & $\begin{array}{l}\text { (Lacrimose mountain tanager; } \\
\text { Anisognathus lacrymosus) }\end{array}$ & 30 & 0 \\
\hline 33 & $\begin{array}{l}\text { Tangara } \\
\text { montana } \\
\text { pechinegra }\end{array}$ & $\begin{array}{l}\text { (Black-chested mountain tanager; } \\
\text { Buthraupis eximia) }\end{array}$ & 12 & 0 \\
\hline 34 & $\begin{array}{l}\text { Tangara } \\
\text { montana } \\
\text { ventriescarlata }\end{array}$ & $\begin{array}{l}\text { (Scarlet-bellied mountain tanager; } \\
\text { Anisognathus lacrymosus) }\end{array}$ & 4 & 0 \\
\hline 35 & $\begin{array}{l}\text { Tapaculo } \\
\text { unicolor }\end{array}$ & $\begin{array}{l}\text { (Unicolored tapaculo; } \\
\text { Scytalopus unicolor) }\end{array}$ & 4 & 0 \\
\hline 36 & $\begin{array}{l}\text { Trogón } \\
\text { enmascarado }\end{array}$ & $\begin{array}{l}\text { (Masked trogon; } \\
\text { Trogon personatus) }\end{array}$ & 3 & 0 \\
\hline 37 & $\begin{array}{l}\text { Tucán andino } \\
\text { pechigris }\end{array}$ & $\begin{array}{l}\text { (Gray-brested mountain tucan; } \\
\text { Andigena hypoglauca) }\end{array}$ & 8 & 0 \\
\hline 38 & $\begin{array}{l}\text { Urraquita } \\
\text { turquesa }\end{array}$ & $\begin{array}{l}\text { (Turquoise jay; } \\
\text { Cyanolyca turcosa) }\end{array}$ & 12 & 0 \\
\hline 39 & $\begin{array}{l}\text { Vencejón } \\
\text { collarejo }\end{array}$ & $\begin{array}{l}\text { (White-collared Swift; } \\
\text { Streptoprocne zonaris) }\end{array}$ & 0 & 14 \\
\hline 40 & $\begin{array}{l}\text { Zamarrito } \\
\text { luciente }\end{array}$ & $\begin{array}{l}\text { (Glowing puffleg; } \\
\text { Eriocnemis vestitus) }\end{array}$ & 20 & 12 \\
\hline
\end{tabular}

